ANNALES

POLONICI MATHEMATICI

$82.1(2003)$

\title{
Geometry of quotient spaces and proximinality
}

\author{
by Yuan Cui (Harbin), Henryk Hudzik (Poznań) and \\ Yaowaluck Khongtham (Chiang Mai)
}

\begin{abstract}
It is proved that if $X$ is a rotund Banach space and $M$ is a closed and proximinal subspace of $X$, then the quotient space $X / M$ is also rotund. It is also shown that if $\Phi$ does not satisfy the $\delta_{2}$-condition, then $h_{\Phi}^{0}$ is not proximinal in $l_{\Phi}^{0}$ and the quotient space $l_{\Phi}^{0} / h_{\Phi}^{0}$ is not rotund (even if $l_{\Phi}^{0}$ is rotund). Weakly nearly uniform convexity and weakly uniform Kadec-Klee property are introduced and it is proved that a Banach space $X$ is weakly nearly uniformly convex if and only if it is reflexive and it has the weakly uniform Kadec-Klee property. It is noted that the quotient space $X / M$ with $X$ and $M$ as above is weakly nearly uniformly convex whenever $X$ is weakly nearly uniformly convex. Criteria for weakly nearly uniform convexity of Orlicz sequence spaces equipped with the Orlicz norm are given.
\end{abstract}

1. Introduction. The notions of nearly uniform convexity (NUC for short) and uniform Kadec-Klee property (UKK) play an important role in some branches of mathematics, for example in fixed point theory (see [1] and [8]). We introduce two new geometric properties of Banach spaces: weakly nearly uniform convexity (WNUC for short) and another property, called the weakly uniform Kadec-Klee property (WUKK). We prove that a Banach space $X$ is WNUC if and only if $X$ is reflexive and it has the WUKK-property.

Let $(X,\|\|)$ be a Banach space and $X^{*}$ be its dual space. By $B(X)$ and $S(X)$ we denote the unit ball and unit sphere of $X$, respectively. Recall that $x \in S(X)$ is said to be an H-point if for any sequence $\left\{x_{n}\right\}$ in $S(X)$ with $x_{n} \stackrel{w}{\rightarrow} x$, we have $\left\|x_{n}-x\right\| \rightarrow 0$. A Banach space $X$ is said to have the Kadec-Klee property (property $\mathrm{H}$ for short) if any point of $S(X)$ is an H-point. A sequence $\left\{x_{n}\right\}$ in $X$ is said to be $\varepsilon$-separated $(\varepsilon>0)$ if $\operatorname{sep}\left(\left\{x_{n}\right\}\right):=\inf \left\{\left\|x_{m}-x_{n}\right\|: m \neq n\right\}>\varepsilon$. Further, $X$ is said to have

2000 Mathematics Subject Classification: 46B20, 46E30, 46E40.

Key words and phrases: quotient space, proximinality, Orlicz sequence space, Orlicz norm, Köthe sequence space, rotundity, weakly uniform Kadec-Klee property, weakly nearly uniform convexity, nearly uniform convexity. 
the uniform Kadec-Klee property (UKK for short) if for any $\varepsilon>0$ there exists $\delta(\varepsilon)>0$ such that if $x$ is a weak limit of an $\varepsilon$-separated sequence in $S(X)$, then $\|x\| \leq 1-\delta(\varepsilon)$. It is well known that Banach spaces with UKK have weakly normal stucture (see [1]). Replacing in the definition of UKK the phrase "for any $\varepsilon>0$ " by "for some $\varepsilon \in(0,1)$ " we define the weakly uniform Kadec-Klee property (WUKK for short). Also the WUKK-property of a Banach space $X$ implies that $X$ has weakly normal structure (the proof remains the same as for UKK).

The notion of nearly uniform convexity of a Banach space was introduced in [11]. It is an infinite-dimensional counterpart of the classical uniform convexity introduced by Clarkson in [3]. Recall that a Banach space $X$ is said to be NUC if for every $\varepsilon>0$ there exists $\delta(\varepsilon)>0$ such that for any sequence $\left\{x_{n}\right\}$ in $B(X)$ with $\operatorname{sep}\left(\left\{x_{n}\right\}\right) \geq \varepsilon$ we have $\operatorname{conv}\left(\left\{x_{n}\right\}\right) \cap \delta B(X) \neq \emptyset$. It is easy to see that NUC implies UKK for any Banach space. Huff [11] has proved that a Banach space $X$ is NUC if and only if it is reflexive and it has UKK. Define a Banach space $X$ to be weakly nearly uniformly convex if there are $\varepsilon \in(0,1)$ and $\delta \in(0,1)$ such that $\operatorname{conv}\left(\left\{x_{n}\right\}\right) \cap \delta B(X) \neq \emptyset$ for every sequence $\left\{x_{n}\right\}$ in $B(X)$ with $\operatorname{sep}\left(\left\{x_{n}\right\}\right) \geq \varepsilon$.

Let $M$ be a closed subspace of a Banach space $X$. We denote by $X / M$ the quotient space of $X$ modulo $M$. It is well known that $X / M$ equipped with the norm $\|[x]\|=\inf \{\|y\|: y \in[x]\}$, where $[x]=\{y \in X: y-x \in M\}$, is also a Banach space. The subspace $M$ of $X$ is called proximinal in $X$ if for any $x \in X$ there is $y \in M$ such that $\|[x]\|=\|x-y\|$.

A point $x \in S(X)$ is said to be an extreme point of $B(X)$ if for any $y, z \in S(X)$ such that $x=(y+z) / 2$ we have $z=y=x$. A Banach space $X$ is called rotund ( $\mathrm{R}$ for short) if any point of $S(X)$ is an extreme point of $B(X)$.

Let $l^{0}$ be the space of all real sequences. A Banach space $X$ is called a Köthe sequence space if $X \subset l^{0}$ and for any $x \in l^{0}$ and $y \in X$ with $|x(i)| \leq|y(i)|$ for all $i \in \mathbb{N}$, we have $x \in X$ and $\|x\| \leq\|y\|$ (see [18]). Since we are interested in infinite-dimensional Köthe sequence spaces $X$, we may assume without loss of generality that there is an element $x \in X$ with $x(i)>0$ for any $i \in \mathbb{N}$.

For any $x \in l^{0}$ we denote by $x^{(n)}$ the element

$$
\overbrace{0, \ldots, 0}^{n \text { times }}, x(n+1), x(n+2), \ldots) .
$$

We say that $x$ is absolutely continuous if $x^{(n)} \rightarrow 0$ in $X$. We denote by $X_{\mathrm{a}}$ the space of all absolutely continuous elements in $X$. A Köthe sequence space $X$ is said to be absolutely continuous if $X_{\mathrm{a}}=X$. We say that $X$ has the semi-Fatou property if for any sequence $\left\{x_{n}\right\}$ in $X$ and any $x \in X$ satisfying $\left|x_{n}(i)\right| \uparrow|x(i)|$ for all $i \in \mathbb{N}$, we have $\left\|x_{n}\right\| \rightarrow\|x\|$. 
A mapping $\Phi: \mathbb{R} \rightarrow[0, \infty)$ is said to be an Orlicz function if $\Phi$ vanishes only at zero, is even and convex.

Given any Orlicz function $\Phi$ we define on $l^{0}$ the convex modular $I_{\Phi}$ by

$$
I_{\Phi}(x)=\sum_{i=1}^{\infty} \Phi(x(i)) .
$$

The linear space $l_{\Phi}$ defined by

$$
l_{\Phi}=\left\{x \in l^{0}: I_{\Phi}(k x)<\infty \text { for some } k>0\right\}
$$

is called the Orlicz space generated by $\Phi$ (see [2] and [12]-[17]). We consider $l_{\Phi}$ equipped with the Amemiya-Orlicz norm

$$
\|x\|^{0}=\inf \left\{\frac{1}{k}\left(1+I_{\Phi}(k x)\right): k>0\right\} .
$$

To simplify notation we write $l_{\Phi}^{0}$ in place of $\left(l_{\Phi}^{0},\|\|^{0}\right)$. The Luxemburg norm in $l_{\Phi}$ is defined by

$$
\|x\|=\inf \left\{\lambda>0: I_{\Phi}(x / \lambda) \leq 1\right\} .
$$

For any $x$ in $l_{\Phi}^{0} \backslash\{0\}$ the set of all numbers $k>0$ such that $\|x\|^{0}=$ $k^{-1}\left(1+I_{\Phi}(k x)\right)$ is denoted by $K(x)$. It is well known (see [2], [16] and [19]) that $K(x)=\left[k_{x}^{*}, k_{x}^{* *}\right]$, where $k_{x}^{*}=\inf \left\{k>0: I_{\Psi}(p \circ k|x|) \geq 1\right\}$ and $k_{x}^{* *}=\sup \left\{k>0: I_{\Psi}(p \circ k|x|) \leq 1\right\}$ if $k_{x}^{*}<\infty$ and $K(x)=\emptyset$ if $k_{x}^{*}=\infty$. Here $\Psi$ denotes the function complementary to $\Phi$ in the sense of Young and $p$ denotes the right derivative of $\Phi$. It is also known that if $\Phi$ satisfies condition $\left(\infty_{1}\right): \Phi(u) / u \rightarrow \infty$ as $u \rightarrow \infty$, then $K(x) \neq \emptyset$ for any $x \in l_{\Phi} \backslash\{0\}$.

We say an Orlicz function $\Phi$ satisfies the $\delta_{2}$-condition $\left(\Phi \in \delta_{2}\right.$ for short) if there are $k \geq 2$ and $u_{0}>0$ such that $\Phi(2 u) \leq k \Phi(u)$ whenever $|u| \leq u_{0}$. In what follows, $h_{\Phi}$ denotes the space of all $x \in l^{0}$ with $I_{\Phi}(k x)<\infty$ for all $k>0$. It is easy to see that $h_{\Phi}=\left(l_{\Phi}\right)_{\mathrm{a}}$. We write briefly $h_{\Phi}^{0}$ in place of $\left(h_{\Phi},\|\|^{0}\right)$. It is well known that $h_{\Phi}=l_{\Phi}$ if and only if $\Phi \in \delta_{2}$ (see [2] and $[17])$.

2. Some results on geometry of quotient spaces. Let us begin with the obvious observation that $\|[x]\|=d(x, M)$, where $d(x, M)$ denotes the distance of $x$ from $M$. Next, we make the following easy remark.

REMARK 1. If $X$ is a Banach space and $M$ is its closed and proximinal subspace, then there is $z \in S(X)$ such that $\|[z]\|=1$.

This follows from Godini's result [7] stating that $M$ is proximinal in $X$ if and only if $q(B(X))=B(X / M)$, where $q$ is the canonical map $X \ni x \mapsto$ $q(x):=[x] \in X / M$. However, for completeness we present a short proof. 
Proof. Take $x \in X \backslash M$. By the proximinality of $M$ in $X$ there is $y \in M$ such that $\|x-y\|=d(x, M)$. Define $z=(x-y) /\|x-y\|$. Then $\|z\|=1$ and

$$
\|[z]\|=\frac{1}{\|x-y\|}\|[x-y]\|=\frac{\|[x]\|}{\|x-y\|}=\frac{\|x-y\|}{\|x-y\|}=1 .
$$

EXAmple 1. Denote by $C[0,1]$ the space of all continuous real functions on the interval $[0,1]$ equipped with the norm $\|x\|_{\infty}=\sup _{0 \leq x \leq 1}|x(t)|$. Define $X=\{x \in C[0,1]: x(0)=0\}$ and $M=\left\{x \in X: \int_{0}^{1} x(t) d t=0\right\}$. It is obvious that $X$ is a Banach space and $M$ is its closed subspace. It is known (see [18, pp. 64-65]) that there is no point on the unit sphere in $X$ with unit distance from $M$. Therefore, by Remark $1, M$ is not proximinal in $X$.

Theorem 1. If $X$ is a rotund Banach space and $M$ is its closed and proximinal subspace, then the Banach space $X / M$ is also rotund.

Proof. Let $[x],[y] \in S(X / M),[x] \neq[y]$, i.e. $x-y \notin M$. By the proximinality of $M$ in $X$, there are $x^{\prime} \in[x]$ and $y^{\prime} \in[y]$ such that $\|[x]\|=\left\|x^{\prime}\right\|=$ $\left\|y^{\prime}\right\|=\|[y]\|=1$. It follows from $[x] \neq[y]$ that $x^{\prime} \neq y^{\prime}$. Since $X$ is rotund we get $\left\|\frac{x^{\prime}+y^{\prime}}{2}\right\|<1$, whence

$$
\left\|\frac{[x]+[y]}{2}\right\|=\left\|\left[\frac{x+y}{2}\right]\right\| \leq\left\|\frac{x^{\prime}+y^{\prime}}{2}\right\|<1,
$$

which means that $X / M$ is rotund.

The next theorem shows that the assumption in Theorem 1 that $M$ is proximinal is essential in general.

THeOREM 2. If $\Phi$ is an Orlicz function vanishing only at zero such that $\Phi \notin \delta_{2}$ and $\Phi$ satisfies condition $\left(\infty_{1}\right)$, then $h_{\Phi}^{0}$ is not proximinal in $l_{\Phi}^{0}$ and $l_{\Phi}^{0} / h_{\Phi}^{0}$ is not rotund.

Proof. It is well known that the spaces $l_{\Phi} / h_{\Phi}$ and $l_{\Phi}^{0} / h_{\Phi}^{0}$ are isometric under the identity map (see [2] and [17]). Recall (see [2]) that

$$
\|x\|<\|x\|^{0} \quad \text { for any } x \in l_{\Phi} \backslash\{0\} .
$$

Assume that $h_{\Phi}^{0}$ is proximinal in $l_{\Phi}^{0}$ and take any $x \in l_{\Phi}^{0} \backslash h_{\Phi}^{0}$. We can find $y \in h_{\Phi}^{0}$ such that $\|[x]\|=\|x-y\|^{0}$. Hence,

$$
\|x-y\|^{0}=\|[x]\| \leq\|x-y\|,
$$

which yields $\|x-y\|^{0}=\|x-y\|$, contradicting (1).

Now, we show that $l_{\Phi}^{0} / h_{\Phi}^{0}$ is not rotund if $\Phi \notin \delta_{2}$ (even if $\Phi$ is strictly convex). It is well known (see [2]) that if $\Phi \notin \delta_{2}$, then one can find $x, y \in l_{\Phi}^{0}$ such that $\operatorname{supp} x \subset\{2 k-1: k \in \mathbb{N}\}$, supp $y \subset\{2 k: k \in \mathbb{N}\}, I_{\Phi}(x) \leq 1 / 2$, $I_{\Phi}(y) \leq 1 / 2$ and $I_{\Phi}(\lambda x)=I_{\Phi}(\lambda y)=\infty$ for all $\lambda>1$. Defining $w=x+y$ and $z=x-y$, we have $\|[w]\|=\|[z]\|=1$, because it is well known (see 
again [2]) that $\|[w]\|=\inf \left\{\lambda>0: I_{\Phi}(w / \lambda)<\infty\right\}$. We have

$$
[x]=\left[\frac{w+z}{2}\right]=\frac{[w]+[z]}{2}
$$

and so $\|[x]\|=1$. This completes the proof that $l_{\Phi}^{0} / h_{\Phi}^{0}$ is not rotund.

REMARK 2. It is well known (see [4]) that if $\Phi$ is an Orlicz function satisfying condition $\left(\infty_{1}\right)$, then $l_{\Phi}^{0}$ is rotund if and only if $\Phi$ is strictly convex on the interval $\left[0, \Pi_{\Phi}(1)\right]$ with $\Pi_{\Phi}(1)=\inf \{t>0: \Psi(p(t)) \geq 1\}$, where $\Psi$ denotes the function complementary to $\Phi$ in the sense of Young and $p$ denotes the right derivative of $\Phi$ on $\mathbb{R}_{+}$. Therefore, taking an Orlicz function $\Phi$ which is strictly convex on the real line and $\Phi \notin \delta_{2}$, we find that $l_{\Phi}^{0}$ is rotund, $h_{\Phi}^{0}$ is not proximinal in $l_{\Phi}^{0}$ (see [9]), and consequently, the quotient space $l_{\Phi}^{0} / h_{\Phi}^{0}$ is not rotund. This explains the role of the proximinality of $M$ in $X$ in Theorem 1.

As we will see below, the situation for weakly nearly uniform convexity is different than for rotundity. However, we first need the following result.

Theorem 3. A Banach space $X$ is weakly nearly uniformly convex if and only if it is reflexive and has the weakly uniform Kadec-Klee property.

Proof. Necessity. We first prove that $X$ is reflexive whenever it is WNUC. Assume that, on the contrary, $X$ is WNUC but nonreflexive. Then by the James Theorem (see [6]) there exist $\left\{x_{n}\right\}$ in $S(X)$ and $\left\{x_{n}^{*}\right\}$ in $S\left(X^{*}\right)$ such that

$$
x_{i}^{*}\left(x_{j}\right)= \begin{cases}\theta & \text { if } i \leq j, \\ 0 & \text { if } i>j,\end{cases}
$$

where $\theta \in(\max \{\varepsilon, \delta\}, 1)$, with $\varepsilon$ arbitrary in $(0,1)$, and $\delta$ from the definition of WNUC. Hence, for $n>m$ we get

$$
\left\|x_{n}-x_{m}\right\| \geq x_{n}^{*}\left(x_{n}-x_{m}\right)=x_{n}^{*}\left(x_{n}\right)=\theta \geq \varepsilon,
$$

which gives $\operatorname{sep}\left(\left\{x_{n}\right\}\right) \geq \varepsilon$. But for any $x \in \operatorname{conv}\left(\left\{x_{n}\right\}\right)$ we have

$$
\|x\|=\left\|\sum_{i=1}^{m} \lambda_{i} x_{i}\right\| \geq x_{m}^{*}\left(\sum_{i=1}^{m} \lambda_{i} x_{i}\right)=\sum_{i=1}^{m} \lambda_{i} x_{m}^{*}\left(x_{i}\right)=\sum_{i=1}^{m} \lambda_{i} \theta=\theta>\delta
$$

for some $m \in \mathbb{N}$ and every $\lambda_{i} \geq 0(i=1, \ldots, m)$ with $\sum_{i=1}^{m} \lambda_{i}=1$. This contradicts the condition $\operatorname{conv}\left(\left\{x_{n}\right\}\right) \cap \delta B(X) \neq \emptyset$ from the definition of WNUC.

Let us prove that $X$ has WUKK whenever it is WNUC. Assume that $X \in$ WNUC, $\left\{x_{n}\right\}$ is a sequence in $B(X)$ with $\operatorname{sep}\left(\left\{x_{n}\right\}\right) \geq \varepsilon, x \in B(X)$ and $x_{n} \stackrel{w}{\rightarrow} x$ as $n \rightarrow \infty$. We have $\operatorname{sep}\left(\left\{x_{n}\right\}_{n=m}^{\infty}\right) \geq \operatorname{sep}\left(\left\{x_{n}\right\}\right) \geq \varepsilon$ for all $m \in \mathbb{N}$. Fix $m=1$. There is $y_{1} \in \operatorname{conv}\left(\left\{x_{n}\right\}_{n=m}^{\infty}\right) \cap \delta B(X)$, i.e. there are $m_{1} \in \mathbb{N}$ and $\lambda_{i} \geq 0$ for $i=1, \ldots, m_{1}$ such that $\sum_{i=1}^{m_{1}} \lambda_{i}=1$ and $y_{1}:=\sum_{i=1}^{m_{1}} \lambda_{i} x_{i}$ satisfies $\left\|y_{1}\right\| \leq \delta$. Fix $m=m_{1}+1$. There is $y_{2} \in \operatorname{conv}\left(\left\{x_{n}\right\}_{n=m_{1}+1}^{\infty}\right) \cap \delta B(X)$, i.e. 
there are $m_{2} \in \mathbb{N}, m_{2} \geq m_{1}+1$ and $\lambda_{i} \geq 0$ for $i=m_{1}+1, \ldots, m_{2}$ with $\sum_{i=m_{1}+1}^{m_{2}} \lambda_{i}=1$ such that $y_{2}=\sum_{i=1}^{m_{2}} \lambda_{i} x_{i}$ satisfies $\left\|y_{2}\right\| \leq \delta$. In such a way we get, by induction, a sequence $\left\{y_{n}\right\}$ in $B(X) \cap \operatorname{conv}\left(\left\{x_{n}\right\}\right)$ of the form

$$
y_{n}=\sum_{i=m_{n}+1}^{m_{n+1}} \lambda_{i} x_{i}, \quad m_{n+1} \geq m_{n}+1 \quad(n=1,2, \ldots)
$$

with $\left\|y_{n}\right\| \leq \delta$ for all $n \in \mathbb{N}$. Since

$$
x^{*}\left(y_{n}\right)=\sum_{i=m_{n}+1}^{m_{n+1}} \lambda_{i} x^{*}\left(x_{i}\right) \rightarrow \sum_{i=m_{n}+1}^{m_{n+1}} \lambda_{i} x^{*}(x)=x^{*}(x) \quad \text { as } n \rightarrow \infty
$$

for any $x^{*} \in X^{*}$, and $\left\|y_{n}\right\| \leq \delta$ for all $n \in \mathbb{N}$, we get $\|x\| \leq \delta$. Therefore $X$ has the weakly uniform Kadec-Klee property.

Sufficiency. Since $X$ has WUKK, there exist $\varepsilon \in(0,1)$ and $\delta \in(0,1)$ such that for every sequence $\left\{x_{n}\right\}$ with $\operatorname{sep}\left(\left\{x_{n}\right\}\right) \geq \varepsilon$ and $x \in B(X)$ such that $x_{n} \stackrel{w}{\rightarrow} x$, we have $\|x\| \leq \delta$.

Assume that $\left\{x_{n}\right\}$ is a sequence in $B(X)$ with $\operatorname{sep}\left(\left\{x_{n}\right\}\right) \geq \varepsilon$. By the reflexivity of $X$ there exist a subsequence $\left\{x_{n_{i}}\right\}$ of $\left\{x_{n}\right\}$ and $x \in B(X)$ such that $x_{n_{i}} \stackrel{w}{\rightarrow} x$. It is obvious that $\operatorname{sep}\left(\left\{x_{n_{i}}\right\}\right) \geq \operatorname{sep}\left(\left\{x_{n}\right\}\right) \geq \varepsilon$. Hence $\|x\| \leq \delta$. Since $x_{n_{i}} \stackrel{w}{\rightarrow} x$, we see that if $\left\{y_{n}\right\}$ is a sequence in $\operatorname{conv}\left(\left\{x_{n_{1}}\right\}\right)$, then $y_{n} \stackrel{w}{\rightarrow} x$. By the Mazur theorem we conclude that $x \in \operatorname{conv}\left(\left\{x_{n}\right\}\right) \cap \delta B(X)$, which means that $X$ is NUC. This finishes the proof of the theorem.

THEOREM 4. If a Banach space $X$ is weakly nearly uniformly convex and $M$ is a closed subspace of $X$, then $X / M$ has the weakly uniform Kadec-Klee property, and $X / M$ is reflexive, so nearly uniformly convex as well.

Proof. By Theorem 3, $X$ is reflexive. So $X / M$ is also reflexive, and to prove that it is WNUC, we need only show that $X / M$ has WUKK. Since $X$ has WUKK, there exist $\varepsilon \in(0,1)$ and $\delta \in(0,1)$ such that if $\left\{x_{n}\right\}$ is a sequence in $B(X)$ with $\operatorname{sep}\left(\left\{x_{n}\right\}\right) \geq \varepsilon$ and $x_{n} \stackrel{w}{\rightarrow} x \in B(X)$, then $\|x\| \leq \delta$. Assume that $\left\{\left[x_{n}\right]\right\}$ is a sequence in $B(X / M)$ with $\operatorname{sep}\left(\left\{\left[x_{n}\right]\right\}\right) \geq \varepsilon$ and $x \in B(X / M)$ is such that $\left[x_{n}\right] \stackrel{w}{\rightarrow}[x]$. Since $X$ is reflexive, there is $x^{\prime} \in B(X)$ such that $x_{n_{i}} \stackrel{w}{\rightarrow} x^{\prime}$. Therefore $\left[x^{\prime}\right]=[x]$, which shows that $x_{n_{i}} \stackrel{w}{\rightarrow} x$. By the double subsequence theorem, we get $x_{n} \stackrel{w}{\rightarrow} x$. Note that $\operatorname{sep}\left(\left\{x_{n}\right\}\right) \geq$ $\operatorname{sep}\left(\left\{\left[x_{n}\right]\right\}\right) \geq \varepsilon$. By the assumption that $X \in$ WNUC we get $\|x\| \leq \delta$, whence $\|[x]\| \leq\|x\| \leq \delta$, which shows that $X / M$ is WNUC.

TheOREM 5. If $X$ is a Köthe sequence space with the semi-Fatou property and the weakly uniform Kadec-Klee property, then $X$ is absolutely continuous.

Proof. Since $X$ has WUKK there are $\varepsilon, \delta \in(0,1)$ such that for every sequence $\left\{x_{n}\right\}$ in $B(X)$ with $\operatorname{sep}\left(\left\{x_{n}\right\}\right) \geq \varepsilon$ and $x_{n} \stackrel{w}{\rightarrow} x \in B(X)$, we have 
$\|x\| \leq \delta$. Suppose that $X$ is not absolutely continuous. Since $X_{\mathrm{a}}$ is a closed subspace of $X$, by the Riesz Lemma, defining $\eta=\max (\varepsilon, \delta)$ one can find $x_{0} \in S(X)$ such that

$$
\left\|x_{0}-y\right\|>\eta^{1 / 2} \quad \text { for all } y \in X_{\mathrm{a}} .
$$

Hence, by the inequality $\eta<\eta^{1 / 2}$, we have

$$
\lim _{n \rightarrow \infty}\left\|(\overbrace{0, \ldots, 0}^{n-1 \text { times }}, x_{0}(n), x_{0}(n+1), \ldots)\right\|>\eta .
$$

Since $X$ has the semi-Fatou property, there is $n_{1} \in \mathbb{N}$ such that

$$
\left\|\sum_{i=1}^{n_{1}} x_{0}(i) e_{i}\right\| \geq \eta
$$

Notice that

$$
\lim _{m \rightarrow \infty}\left\|\sum_{i=n_{1}+1}^{m} x_{0}(i) e_{i}\right\|=\left\|(\overbrace{0, \ldots, 0}^{n_{1} \text { times }}, x_{0}\left(n_{1}+1\right), x_{0}\left(n_{1}+2\right), \ldots)\right\|>\eta .
$$

So, there exists $n_{2}>n_{1}$ such that

$$
\left\|\sum_{i=n_{1}+1}^{n_{2}} x_{0}(i) e_{i}\right\| \geq \eta
$$

In such a way, we get by induction an increasing sequence $\left\{n_{i}\right\}$ of natural numbers such that

$$
\left\|\sum_{i=n_{i}+1}^{n_{i+1}} x_{0}(i) e_{i}\right\| \geq \eta \quad(i=1,2, \ldots) .
$$

Define $x_{i}=\sum_{i=n_{i}+1}^{n_{i+1}} x_{0}(i) e_{i}$. Then

$$
\left\|x_{i}\right\| \geq \eta \quad \text { for all } i \in \mathbb{N} .
$$

We will show that

$$
x_{i} \stackrel{w}{\rightarrow} x_{0} \quad \text { as } i \rightarrow \infty .
$$

It is well known that for any Köthe sequence space (see [12]) we have

$$
X^{*}=X^{\prime} \oplus S,
$$

where $S$ is the space of all singular functionals over $X$, i.e. the functionals which vanish on the subspace $X_{\mathrm{a}}$, and

$$
X^{\prime}=\left\{y \in l^{0}: \sum_{i=1}^{\infty}|x(i) y(i)|<\infty \text { for any } x \in X\right\},
$$


that is, $X^{\prime}$ is the Köthe dual of $X$. This means that every $x^{*} \in X^{*}$ is uniquely represented in the form

$$
x^{*}=T_{y}+\phi,
$$

where $\phi \in S$ and $T_{y}$ is the functional generated by an element $y \in X^{\prime}$ by the formula

$$
T_{y}(x)=\sum_{i=1}^{\infty} x(i) y(i) \quad\left(\forall x=\{x(i)\}_{i=1}^{\infty} \in X\right) .
$$

It is obvious that $\phi\left(x_{i}\right)=0$ for any $\phi \in S$ and all $i \in \mathbb{N}$. Moreover

$$
T_{y}\left(x_{i}\right)=\sum_{j=n_{i}+1}^{n_{i+1}} x_{0}(j) y(j) \rightarrow 0 \quad \text { as } i \rightarrow \infty
$$

because the series $\sum_{j=1}^{\infty} x_{0}(j) y(j)$ converges. This means that $x_{i} \stackrel{w}{\rightarrow} 0$, i.e. condition (3) holds. Defining $z_{i}=x_{0}-x_{i}(i=1,2, \ldots)$, we have $\left\|z_{i}\right\| \leq 1$ for $i=1,2, \ldots, z_{i} \stackrel{w}{\rightarrow} x_{0}, \operatorname{sep}\left(\left\{z_{i}\right\}\right) \geq \sup \left\{\left\|x_{i}\right\|: i \in \mathbb{N}\right\} \geq \eta \geq \varepsilon$ and $x_{0} \in S(X)$, a contradiction finishing the proof.

THEOREM 6. For the Orlicz sequence space $l_{\Phi}^{0}$ the following assertions are equivalent:

(i) $l_{\Phi}^{0}$ has the uniform Kadec-Klee property,

(ii) $l_{\Phi}^{0}$ has the weakly uniform Kadec-Klee property,

(iii) $\Phi \in \delta_{2}$.

Proof. The implication (i) $\Rightarrow$ (ii) is trivial. Since, by Theorem 5 and the fact that $l_{\Phi}^{0}$ has the semi-Fatou property, the weakly uniform Kadec-Klee property implies order continuity (see Theorem 5), and since order continuity of $l_{\Phi}^{0}$ is equivalent to $\Phi \in \delta_{2}$, the implication (ii) $\Rightarrow$ (iii) is true. So, we need only prove the implication (iii) $\Rightarrow$ (i).

We first prove that if $x \in l_{\Phi}^{0} \backslash\{0\}$ and $K(x)=\emptyset$, then $\operatorname{Card}(N(x))<\infty$, where $N(x)=\operatorname{supp} x$, and

$$
\|x\|^{0}=A \sum_{i \in N(x)}|x(i)|,
$$

where $A=\lim _{u \rightarrow \infty} \Phi(u) / u$. Note that if $K(x)=\emptyset$ we must have $A<\infty$. It is clear that if $\operatorname{Card}(N(x))=\infty$, then there exists $k>0$ such that $I_{\Psi}(p \circ k|x|) \geq 1$, i.e. $k_{x}^{*}<\infty$. Hence $K(x) \neq \emptyset$, a contradiction, which proves that $K(x)=\emptyset$ implies that $\operatorname{Card}(N(x))<\infty$. The fact that $K(x)=\emptyset$ yields (4) has been proved in [4].

Take an arbitrary $\varepsilon>0$, any sequence $\left\{x_{n}\right\}$ in $B\left(l_{\Phi}^{0}\right)$ with $\operatorname{sep}\left(\left\{x_{n}\right\}\right)>\varepsilon$ and any $x \in l_{\Phi}^{0}$ with $x_{n} \stackrel{w}{\rightarrow} x$. It is clear that $\operatorname{sep}\left(\left\{\sum_{i=m}^{\infty} x_{n}(i) e_{i}\right\}\right)>\varepsilon$ for any $m \in \mathbb{N}$. Without loss of generality, we may assume that $\left\|\sum_{i=m}^{\infty} x_{n}(i) e_{i}\right\|^{0}>$ 
$\varepsilon / 2$ for all $m \in \mathbb{N}$. Since $\Phi \in \delta_{2}$, there exists $\varepsilon_{1} \in(0, \varepsilon)$ depending only on $\varepsilon$ such that $I_{\Phi}\left(\sum_{i=m}^{\infty} x_{n}(i) e_{i}\right)>\varepsilon_{1}$ for all $m \in \mathbb{N}$.

Take $m$ so large that $\left\|\sum_{i=m}^{\infty} x_{n}(i) e_{i}\right\|^{0}>\|x\|^{0}-\varepsilon_{1} / 4$. Since $x_{n}(i) \rightarrow$ $x(i)$ for $i=1,2, \ldots$, we conclude that there exists $m_{0} \in \mathbb{N}$ such that $\left\|\sum_{i=1}^{m} x_{n}(i) e_{i}\right\|^{0}>\|x\|^{0}-\varepsilon_{1} / 4$ for all $n>m_{0}$.

We divide the remaining part of the proof into two cases.

CASE I: $K\left(x_{n}\right) \neq \emptyset$ for $n=1,2, \ldots$ Then for $k_{n} \in K\left(x_{n}\right)$ we have

$$
\begin{aligned}
1 & \geq\left\|x_{n}\right\|^{0}=\frac{1}{k_{n}}\left(1+\sum_{i=1}^{m} \Phi\left(k_{n} x_{n}(i)\right)+\sum_{i=m+1}^{\infty} \Phi\left(k_{n} x_{n}(i)\right)\right) \\
& =\frac{1}{k_{n}}\left(1+\sum_{i=1}^{m} \Phi\left(k_{n} x_{n}(i)\right)\right)+\frac{1}{k_{n}} \sum_{i=m+1}^{\infty} \Phi\left(k_{n} x_{n}(i)\right) \\
& \geq\left\|\sum_{i=1}^{m} x_{n}(i) e_{i}\right\|^{0}+\sum_{i=m+1}^{\infty} \Phi\left(x_{n}(i)\right)>\|x\|^{0}-\frac{\varepsilon_{1}}{4}+\varepsilon_{1},
\end{aligned}
$$

whence $\|x\|^{0}<1-\frac{3}{4} \varepsilon_{1}$ for $n>m$.

CASE II: $K\left(x_{n}\right)=\emptyset$ for $n=1,2, \ldots$ Then

$$
\begin{aligned}
1 & \geq\left\|x_{n}\right\|^{0}=\left\|\sum_{i=1}^{m} x_{n}(i) e_{i}\right\|^{0}+\left\|\sum_{i=m+1}^{\infty} x_{n}(i) e_{i}\right\|^{0} \\
& >\|x\|^{0}-\frac{\varepsilon_{1}}{4}+\frac{\varepsilon_{1}}{2}=\|x\|^{0}+\frac{\varepsilon_{1}}{4},
\end{aligned}
$$

whence $\|x\|^{0}<1-\varepsilon_{1} / 4$ for all $n>m$.

Notice that it is enough to consider only cases I and II because defining $N_{1}=\left\{n \in \mathbb{N}: K\left(x_{n}\right) \neq \emptyset\right\}$ and $N_{2}=\mathbb{N} \backslash N_{1}$, we have $\operatorname{Card}\left(N_{1}\right)=\infty$ or $\operatorname{Card}\left(N_{2}\right)=\infty$. If $\operatorname{Card}\left(N_{i}\right)=\infty$, we can assume without loss of generality that $N_{i}=\mathbb{N}$. The proof is finished.

Let us recall here that property UKK for Musielak-Orlicz sequence spaces equipped with the Luxemburg norm has been characterized in [5].

\section{References}

[1] J. M. Ayerbe Toledano, T. Domínguez Benavides and G. López Acedo, Measures of Noncompactness in Metric Fixed Point Theory, Oper. Theory Adv. Appl. 99, Birkhäuser, Basel, 1997.

[2] S. T. Chen, Geometry of Orlicz spaces, Dissertationes Math. 356 (1996).

[3] J. A. Clarkson, Uniformly convex spaces, Trans. Amer. Math. Soc. 40 (1936), 396414.

[4] Y. A. Cui, H. Hudzik, M. Nowak and R. Płuciennik, Some geometric properties in Orlicz sequence spaces equipped with Orlicz norm, J. Convex Anal. 6 (1999), 91-113. 
[5] Y. A. Cui, H. Hudzik and R. Płuciennik, Banach-Saks property in some Banach sequence spaces, Ann. Polon. Math. 65 (1997), 193-202.

[6] J. Diestel, Sequences and Series in Banach Spaces, Grad. Texts in Math. 92, Springer, 1984.

[7] G. Godini, Characterization of proximinal linear subspaces in normed linear spaces, Rev. Roumaine Math. Pures Appl. 18 (1973), 901-906.

[8] K. Goebel and W. A. Kirk, Topics in Metric Fixed Point Theory, Cambridge Univ. Press, 1990.

[9] A. S. Granero and H. Hudzik, The classical Banach spaces $l_{\varphi} / h_{\varphi}$, Proc. Amer. Math. Soc. 124 (1996), 3777-3787.

[10] - - - On some proximinal subspaces of modular spaces, Acta Math. Hungar. 85 (1999), 59-79.

[11] R. Huff, Banach spaces which are nearly uniformly convex, Rocky Mountain J. Math. 10 (1980), 473-479.

[12] L. V. Kantorovich and G. P. Akilov, Functional Analysis, Nauka, Moscow, 1977 (in Russian).

[13] M. A. Krasnosel'skiǔ and Ya. B. Rutickiü, Convex Functions and Orlicz Spaces, Noordhoff, Groningen, 1961 (translation).

[14] W. A. J. Luxemburg, Banach Function Spaces, thesis, Delft, 1955.

[15] L. Maligranda, Orlicz Spaces and Interpolation, Seminars in Math. 5, Campinas, 1989.

[16] J. Musielak, Orlicz Spaces and Modular Spaces, Lecture Notes in Math. 1034, Springer, 1983.

[17] M. M. Rao and Z. D. Ren, Theory of Orlicz Spaces, Dekker, New York, 1991.

[18] A. E. Taylor and D. C. Lay, Introduction to Functional Analysis, 2nd ed., Wiley, New York, 1980.

[19] C. X. Wu and H. Y. Sun, Norm calculation and complex convexity of MusielakOrlicz sequence space, Chin. Ann. Math. Ser. A 12 (1991), suppl., 98-102.

Department of Mathematics

Harbin University of Sciences

and Technology

Harbin, P.R. China

Faculty of Science

Maejo University

Chiang Mai, Thailand
Faculty of Mathematics and Computer Science Adam Mickiewicz University Umultowska 87 61-614 Poznań, Poland E-mail: hudzik@amu.edu.pl 